\title{
Computing on Authenticated Data for Adjustable Predicates
}

\author{
Björn Deiseroth Victoria Fehr Marc Fischlin Manuel Masz \\ Nils Fabian Reimers Richard Stein \\ Darmstadt University of Technology, Germany
}

\begin{abstract}
The notion of P-homomorphic signatures, introduced by Ahn et al. (TCC 2012), generalizes various approaches for public computations on authenticated data. For a given predicate $\mathrm{P}$ anyone can derive a signature for a message $m^{\prime}$ from the signatures of a set of messages $M$, as long as $\mathrm{P}\left(M, m^{\prime}\right)=1$. This definition hence comprises notions and constructions for concrete predicates $\mathrm{P}$ such as homomorphic signatures and redactable signatures.

In our work we address the question of how to combine $\mathrm{P}_{i}$-homomorphic schemes for different predicates $\mathrm{P}_{1}, \mathrm{P}_{2}, \ldots$ to create a richer and more flexible class of supported predicates. One approach is to statically combine schemes for predicates into new schemes for logical formulas over the predicates, such as a scheme for AND $\left(\mathrm{P}_{1} \wedge \mathrm{P}_{2}\right)$. The other approach for more flexibility is to derive schemes which allow the signer to dynamically decide which predicate to use when signing a message, instead of supporting only a single, fixed predicate.

We present two main results. One is to show that one can indeed devise solutions for the static combination for AND, and for dynamically adjustable solutions for choosing the predicate on the fly. Moreover, our constructions are practical and add only a negligible overhead. The other main result is an impossibility result for static combinations. Namely, we prove that, in contrast to the case of AND, many other formulas like the logical OR $\left(P_{1} \vee P_{2}\right)$ and the NOT $(\neg P)$ do not admit generic combinations through so-called canonical constructions. This implies that one cannot rely on general constructions in these cases, but must use other methods instead, like finding new predicate-specific solutions from scratch.
\end{abstract}

\section{Introduction}

The notion of P-homomorphic signatures has been put forward by $\mathrm{Ahn}$ et al. $\mathrm{ABC}^{+} 12$ as a generalization of several concurrent approaches to compute on authenticated data. The predicate $\mathrm{P}$ takes as input a set of messages $M$ and determines the admissible messages $m^{\prime}$ which can be derived from $M$, and for which a signature can be publicly computed from the signatures for the messages in $M$. Examples covered by such signatures include homomorphic signatures Des93, JMSW02, CJL09, BFKW09, GKKR10, JWL11, AL11, BF11b, BF11a, Fre12, CFW12 where $m^{\prime}$ is the sum of all messages in $M$, transitive signatures [MR02, BN02, SSM04, Yi07, $\mathrm{WCZ}^{+} 07, \mathrm{CH} 12$. where $m^{\prime}$ describes a path in a graph given by $M$, and redactable signatures [JMSW02, SBZ01, $\mathrm{MSI}^{+}$03, $\mathrm{ACdMT05}, \mathrm{HHH}^{+}$08, CLX09, NTKK09, $\left.\mathrm{BBD}^{+} 10\right]$ where $m^{\prime}$ is a substring of the single message $M$.

Ahn et al. $\mathrm{ABC}^{+} 12$ proposed two general security notions for P-homomorphic signatures. The first one is unforgeability and says that one should not be able to forge signatures for fresh messages which have not been signed before, and which are not publicly derivable. The other 
notion is called context hiding and provides strong privacy. It says that a derived signature for an admissible message $m^{\prime}$ and freshly created signatures for $m^{\prime}$ have statistically close distributions. This guarantees for instance that the original message in case of redactable signatures remains hidden. The context hiding notion has been subsequently refined in [ALP12].

P-Homomorphic Signatures with Adjustable Predicates. While the abstract notion of Phomomorphic signatures is very handy for arguing about the security of solutions, any construction so far, even the ones in $\mathrm{ABC}^{+} 12$, $\mathrm{ALP12}$, are for a specific fixed predicate $\mathrm{P}$, such as quoting substrings of a message. What is currently unknown is how to adjust solutions for fixed predicates in the following sense:

- One desirable option may be the possibility to combine a set of given homomorphic schemes for predicates $\mathrm{P}_{1}, \mathrm{P}_{2}, \ldots$ into one for a new $\mathrm{P}$-homomorphic signature scheme. Here, $\mathrm{P}$ may be a simple combination such as $\mathrm{P}_{1} \wedge \mathrm{P}_{2}$ or $\mathrm{P}_{1} \vee \mathrm{P}_{2}$, or describe even more complex functions. An example are two redactable schemes, one allowing for redaction only at the front of the message $\left(P_{1}\right)$, and the other one enabling redaction only at the end $\left(P_{2}\right)$. Then a $P_{1} \vee P_{2^{-}}$ homomorphic scheme would be a scheme for quoting substrings, by first pruning at the front and then truncating in another step at the end. Note that the problem here is to present a general transformation which supports a rich set of combinations from, say, basic predicates $\mathrm{P}_{1}, \mathrm{P}_{2}, \ldots$, instead of having to build schemes for $\mathrm{P}$ from scratch.

- Another desirable feature, which is not offered by the previous ability to combine predicates, is that signer can decide "on the fly" for each signature which predicate $\mathrm{P}$ the signature should support. Here, the set of admissible predicates is only bound by the universe $\mathcal{P}$ of predicates for which such signature schemes have been devised yet. This would allow to make the set of admissible message derivates depend on the message itself, e.g., supporting selective redaction for different messages.

We call general constructions with the first property statically adjustable because the combined predicate $\mathrm{P}$ is fixed at the time of key generation. The latter schemes are called dynamically adjustable. Both approaches have their merits and display their full power only in combination. One can first derive (statically) adjustable schemes for a larger universe $\mathcal{P}$, and then use this universe for the dynamically adjustable scheme.

Constructing Schemes with Statically Adjustable Predicates. We first investigate simple static combinations such as $P_{1} \wedge P_{2}, P_{1} \vee P_{2}$, and $\neg P$. Having solutions for these cases would immediately allow arbitrarily complex combinations of predicates. Our first result is to confirm for the logical AND that the "componentwise" solution works: sign each message with the schemes for predicates $\mathrm{P}_{1}, \mathrm{P}_{2}$ individually, and derive signatures by applying the corresponding algorithms for each component.

Our main result is to show that the logical OR, $\mathrm{P}_{1} \vee \mathrm{P}_{2}$, in general does not admit canonical constructions. Such canonical constructions can combine given signatures of the individual schemes into one for the $\mathrm{P}_{1} \vee \mathrm{P}_{2}$ predicate, and can vice versa split any signature for the OR into parts for the individual schemes. Our AND construction is of this type. Our negative result for the OR holds for (almost) arbitrary predicates $\mathrm{P}_{1}, \mathrm{P}_{2}$, essentially only excluding trivial examples like $\mathrm{P}_{1} \vee \mathrm{P}_{1}$. Note that we cannot hope to show a similar result for non-canonical solutions, as for some cases we know constructions from scratch for $\mathrm{P}_{1} \vee \mathrm{P}_{2}$ (e.g., for quotable substrings).

We actually present a more general result, saying that one cannot find canonical constructions for any predicate combination $f\left(\mathrm{P}_{1}, \mathrm{P}_{2}, \ldots\right)$ if one is able to efficiently find a derivable message 
$m^{\prime}$ under $f\left(\mathrm{P}_{1}, \mathrm{P}_{2}, \ldots\right)$ and from a message set $M$, such that $m^{\prime}$ is not derivable under one of the predicates individually. This excludes the AND case, because any derivable message $m^{\prime}$ in $P_{1} \wedge P_{2}$ must be also valid according to both in $\mathrm{P}_{1}$ and $\mathrm{P}_{2}$. Yet, this notion includes the OR case if $m^{\prime}$ can be derived under one predicate, and therefore the OR, but not under the other predicate. It also covers the NOT case straightforwardly, because if $m^{\prime}$ is derivable under $f\left(\mathrm{P}_{1}\right)=\neg \mathrm{P}_{1}$, then it is clearly not derivable under $\mathrm{P}_{1}$. The impossibility result holds even if the canonical construction depends on $f$ and the predicates. Put differently, it seems that the only general and non-trivial solutions for statically adjustable predicates are the ones for logical ANDs.

Constructing Schemes with Dynamically Adjustable Predicates. Does the negative result for statically adjustable parameters also rule out solutions for the dynamic case? Not necessarily, because in this case we assume that the signer adaptively chooses the predicate $\mathrm{P}$ from the universe $\mathcal{P}$ for which constructions are already known. Indeed we show that the "certify-then-sign" construction provides a solution in this case: use a regular signature scheme to certify a public key for the $\mathrm{P}$-homomorphic scheme for the chosen predicate $\mathrm{P} \in \mathcal{P}$ and sign the message under the secret key for $\mathrm{P}$. Some care must be taken, though, because in order to preserve context hiding the key pair for the P-homomorphic scheme must remain fixed throughout the life time.

\section{Preliminaries}

We recall the definition and security notions of $\mathrm{P}$-homomorphic signatures, as given in $\mathrm{ABC}^{+} 12$, ALP12, and adopt them slightly for our adjustable setting.

\subsection{Adjustable $\mathcal{P}$-homomorphic Signature Schemes}

We assume a fixed but public universe $\mathcal{P}$ of predicates $\mathrm{P}_{1}, \mathrm{P}_{2}, \ldots$, each predicate associated with a publicly known $\mathrm{P}_{i}$-homomorphic signature scheme. A predicate $\mathrm{P}_{i}: 2^{\mathcal{M}} \times \mathcal{M} \rightarrow\{0,1\}$ indicates whether a set of messages $M$ allows to derive another message $m^{\prime}$ from the message space $\mathcal{M}$ or not. We give the signer and the verifier the predicate $P$ in question as additional input. In case of a single fixed predicate $P$, as for the statically adjustable setting, where the universe $\mathcal{P}$ is a singleton, this is an invariant for the scheme and could be ignored by both algorithms. In fact, in this case the notion basically coincides with the definition of a P-homomorphic scheme, the only difference being the predicate given to the signers and verifier as additional input. In this sense the definition of schemes with statically adjustable predicates is a rehash of the notion of P-homomorphic signatures. We stress that we do not suggest to change the terminology for P-homomorphic schemes. The reader should bear in mind, however, that schemes with statically adjustable predicates in this paper implicitly assume a construction from selected P-homomorphic schemes underneath. In light of this it matches the dynamic counterpart where predicates are chosen adaptively for each signature.

We simplify the notation below, and write $\operatorname{Verify}(p k, M, \Sigma, \mathrm{P})$ as shorthand for $\bigwedge_{m \in M} \operatorname{Verify}\left(p k, m, \sigma_{m}, \mathrm{P}\right)$ with $\Sigma=\left\{\sigma_{m}\right\}_{m \in M}$. Similarly, we sometimes write $\Sigma \leftarrow \operatorname{Sign}(s k, M, \mathrm{P})$ for $\Sigma=\{\operatorname{Sign}(s k, m, \mathrm{P}) \mid m \in M\}$.

Definition 2.1 (Adjustable $\mathcal{P}$-homomorphic Signature Scheme) $A$ (statically or dynamically) adjustable $\mathcal{P}$-homomorphic signature scheme is a tuple of PPT algorithms (KeyGen, Sign, SignDer, Verify) such that:

- $(s k, p k) \leftarrow K e y G e n\left(1^{\lambda}\right)$ maps the security parameter $\lambda \in \mathbb{N}$, given in unary, to a key pair. 
- $\sigma \leftarrow \operatorname{Sign}(s k, m, P)$ on input the secret key sk, a message $m \in \mathcal{M}$, and a predicate $P \in \mathcal{P}$ returns a signature $\sigma$ to $m$ and $P$.

- $\sigma^{\prime} \leftarrow \operatorname{SignDer}\left(p k, M, \Sigma, m^{\prime}, P\right)$ takes as input the public key $p k$, a set of messages $M \subseteq \mathcal{M}$ along with signatures $\Sigma=\left\{\sigma_{m}\right\}_{m \in M}$, a message $m^{\prime} \in \mathcal{M}$, and the predicate $P \in \mathcal{P}$ to be applied, and outputs a signature $\sigma^{\prime}$ (or a special symbol $\perp$ indicating failure).

- $b \leftarrow \operatorname{Verify}(p k, m, \sigma, P)$, given the public key $p k$, a signature $\sigma$, a message $m \in \mathcal{M}$, and a predicate $P \in \mathcal{P}$, returns 1 if the signature is valid for the given message, and 0 if not.

We assume the usual correctness condition, namely, that for any $\lambda \in \mathbb{N}$, any $(s k, p k) \leftarrow$ KeyGen $\left(1^{\lambda}\right)$, any $\left(m, M, m^{\prime}\right) \in \mathcal{M} \times 2^{\mathcal{M}} \times \mathcal{M}$ and any $P \in \mathcal{P}$ we have:

- if $\sigma \leftarrow \operatorname{Sign}(s k, m, P)$, then $\operatorname{Verify}(p k, m, \sigma, P)=1$ with probability 1 ; and

- for any $\Sigma=\left\{\sigma_{m}\right\}_{m \in M}$, if Verify $(p k, M, \Sigma, P)=1$ and $P\left(M, m^{\prime}\right)=1$, then for any $\sigma^{\prime} \leftarrow$ SignDer $\left(p k, M, \Sigma, m^{\prime}, P\right)$ we have Verify $\left(p k, m^{\prime}, \sigma^{\prime}, P\right)=1$ with probability 1 .

\subsection{Unforgeability}

For any predicate $\mathrm{P}$ and set $M$ of messages it is convenient to consider the set of messages which can be derived (recursively) from $M$ through $P$. Hence, similar to $\mathrm{ABC}^{+} 12$, we define $\mathrm{P}(M)=$ $\left\{m^{\prime} \in \mathcal{M} \mid \mathrm{P}\left(M, m^{\prime}\right)=1\right\}$ for any $M \subseteq \mathcal{M}$, as well as $\mathrm{P}^{0}(M)=M$ and $\mathrm{P}^{i}(M)=\mathrm{P}\left(\mathrm{P}^{i-1}(M)\right)$ for $i>0$. Let $\mathrm{P}^{*}(M)=\bigcup_{i \in \mathbb{N}_{0}} \mathrm{P}^{i}(M)$. We sometimes switch between the set $\mathrm{P}^{*}(M)$ and its predicate analogue, with $\mathrm{P}^{*}\left(M, m^{\prime}\right)=1$ iff $m^{\prime} \in \mathrm{P}^{*}(M)$. Unless mentioned differently, we assume that any predicate can be evaluated efficiently.

We also presume, without further mentioning it, that predicates are monotone, that is, $\mathrm{P}\left(M^{\prime}\right) \subseteq$ $\mathrm{P}(M)$ if $M^{\prime} \subseteq M$. It follows inductively that $\mathrm{P}^{*}\left(M^{\prime}\right) \subseteq \mathrm{P}^{*}(M)$ in this case as well. This is necessary to ensure that, below in the unforgeability game, the set of messages for which a signature can be trivially derived from known signatures for $M$, does not shrink by asking for more signatures 1 An alternative is to consider below all subsets $M^{\prime} \subseteq M$ and declare that any message which is in $\mathrm{P}\left(M^{\prime}\right)$ to be a message for which a signature is trivial to derive from the signatures for messages in $M^{\prime}$.

We again consider both the static and the dynamic case simultaneously, with the understanding that the predicate is fixed in the static case via $\mathcal{P}=\{P\}$.

Definition 2.2 (Unforgeability) A (statically or dynamically) adjustable $\mathcal{P}$-homomorphic signature scheme (KeyGen, Sign, SignDer, Verify) is called unforgeable, if any PPT adversary $\mathcal{A}$ has a negligible advantage in the following game:

1. The challenger $\mathcal{C}$ generates the key pair $(s k, p k) \leftarrow K e y G e n\left(1^{\lambda}\right)$ and gives pk to the adversary $\mathcal{A}$. The challenger initializes two empty sets $T$ and $Q$.

2. $\mathcal{A}$ interleaves adaptively the following queries:

- Signing queries: $\mathcal{A}$ chooses a message $m \in \mathcal{M}$ and a predicate $P \in \mathcal{P}$, upon which $\mathcal{C}$ returns a unique handle $h$ to $\mathcal{A}$, runs $\sigma \leftarrow \operatorname{Sign}(s k, m, P)$, and stores $(h, m, \sigma, P)$ in $T$.

\footnotetext{
${ }^{1}$ Interestingly, this is not stipulated explicitly in previous works $\mathrm{ABC}^{+} 12$, ALP12. Still, the predicates for the constructions there satisfy this property. It is, nonetheless, generally required for a reasonable definition in order to avoid trivial examples of schemes which are formally unforgeable, but intuitively insecure.
} 
- Derivation queries: $\mathcal{A}$ chooses a set of handles $\boldsymbol{h}=\left\{h_{i}\right\}_{i}$, a message $m^{\prime} \in \mathcal{M}$ and a predicate $P$. The challenger $\mathcal{C}$ retrieves the tuples $\left(h_{i}, m_{i}, \sigma_{i}, P_{i}\right)$ from $T$ and returns $\perp$ if one of these tuples does not exist, $P_{i} \neq P$ for some $i$, or $P\left(M, m^{\prime}\right)=0$. Otherwise, the challenger returns a unique handle $h^{\prime}$ to $\mathcal{A}$, runs $\sigma^{\prime} \leftarrow \operatorname{SignDer}\left(p k, M,\left\{\sigma_{m}\right\}_{m \in M}, m^{\prime}, P\right)$ for $M=\left\{m_{i}\right\}_{i}$ and stores $\left(h^{\prime}, m^{\prime}, \sigma^{\prime}, P\right)$ in $T$.

- Reveal queries: If $\mathcal{A}$ chooses a handle $h$ then $\mathcal{C}$ returns $\perp$ if there does not exist a tuple of the form $(h, m, \sigma, P)$ in $T$. Otherwise, it returns $\sigma$ to $\mathcal{A}$ and adds $(m, \sigma, P)$ to the set $Q$.

3. $\mathcal{A}$ outputs a pair $(m, \sigma, P)$ and wins if the following conditions hold:

- $\operatorname{Verify}(p k, m, \sigma, P)=1$, and

- $m \notin P^{*}\left(M_{P}\right)$, where $M_{P}=\{m \in \mathcal{M} \mid(m, *, P) \in Q\}$, the set of messages in the query set $Q$ for the same predicate $P$.

Note that the condition on $m \notin \mathrm{P}^{*}\left(M_{\mathrm{P}}\right)$ can be relaxed by considering the set $M$ of messages which have been signed under some predicate (and not only those which have been signed under the same predicate $\mathrm{P}$ as in the forgery attempt). In the static case both cases coincide, of course.

\subsection{Context Hiding}

The original definition of $\mathrm{Ahn}$ et al. $\left[\mathrm{ABC}^{+} 12\right.$ requires a strong privacy requirement, basically saying that a derived signature (from previously signed messages $M$ ), and a fresh signature for the new message $m^{\prime}$ are statistically close. It follows that a derived signature does not leak any information about the starting messages $M$, and thus implies other common privacy notions for, say, redactable signature schemes $\left[\mathrm{BBD}^{+} 10\right]$. Still, the notion has been strengthened in [ALP12] to adaptive context hiding and complete context hiding, basically saying that derived signatures (for messages with any valid signatures) and fresh signatures are close. The generalization to valid signatures as input, instead of only signed messages, allows to cover previously excluded cases like rerandomizable signatures.

While the notion of adaptive context hiding is game-based, the notion of complete context hiding is defined through statistically close distributions of signatures. It is convenient for us here to present the latter definition also through a game, but considering unbounded adversaries (as opposed to efficient adversaries for adaptive context hiding). Otherwise the notions are identical. Our game-based definition of complete context hiding can be seen easily to be equivalent to the distributional approach in [ALP12].

Definition 2.3 ((Complete and Adaptive) Context Hiding) A (statically or dynamically) adjustable $\mathcal{P}$-homomorphic signature scheme (KeyGen, Sign, SignDer, Verify) is called completely (resp. adaptively) context hiding, if any unbounded (resp. PPT) adversary $\mathcal{A}$ has a negligible advantage in the following game:

1. The challenger $\mathcal{C}$ generates the key pair $(s k, p k) \leftarrow \operatorname{KeyGen}\left(1^{\lambda}\right)$ and gives $(s k, p k)$ to the adversary $\mathcal{A}$.

2. The adversary selects a set $M$ of messages, and set $\left\{\sigma_{m}\right\}_{m \in M}$ of signatures, a predicate $P \in \mathcal{P}$, and a message $m^{\prime}$ and hands it to the challenger. If $P\left(M, m^{\prime}\right)=0$ or if $\operatorname{Verify}\left(p k, M,\left\{\sigma_{m}\right\}_{m \in M}, P\right)=0$ then the challenger immediately returns $\perp$. Else it picks a random bit $b \leftarrow\{0,1\}$ and computes a derived siganture $\sigma^{\prime} \leftarrow \operatorname{SignDer}\left(p k, M,\left\{\sigma_{m}\right\}_{m \in M}, m^{\prime}, P\right)$ if $b=0$, and a fresh signature $\sigma^{\prime} \leftarrow \operatorname{Sign}\left(s k, m^{\prime}, P\right)$ in case $b=1$. It returns $\sigma^{\prime}$ to the adversary. 
3. Eventually the adversary outputs a bit $b^{*} \in\{0,1\}$ and wins if $b^{*}=b$. The advantage of $\mathcal{A}$ is defined to be $\boldsymbol{A} \boldsymbol{d} \boldsymbol{v}(\mathcal{A})=\left|\operatorname{Prob}\left[b^{*}=b\right]-\frac{1}{2}\right|$.

Some remarks are in place. First note that the adversary can ask the challenger only once. A standard hybrid argument shows that this remains true for multiple (polynomially many) queries for which the challenger re-uses the same bit $b$. For both cases, the static and the dynamic one, the advantage grows by a factor proportional to the number of queries.

Secondly, note that in the dynamically adjustable case we do not aim to hide the predicate $\mathrm{P}$ which has been used to compute the signature. In a stronger requirement one could demand that the actual predicate remains hidden, either among all predicates from the universe, or among the predicates for which the public derivation algorithm would succeed. The former would require a super-polynomial set $\mathcal{P}$ (else the privacy attacker could probe the derivation algorithms for all predicates). The latter would mean a trade-off between privacy, usability, and the signer's intention for restricting the class of admissible public operations: if the signature would hide the corresponding predicate among multiple possibilities, then signatures for a different predicate than the original choice may be derivable. This would imply that the signer loses some control about the (in)ability to derive further signatures. Hence, we do not pursue such stronger requirements here.

\section{$3 \quad$ Statically Adjustable Computations}

In this section we investigate statically adjustable constructions for the basic operations AND, OR, and NOT. As explained in the introduction, we can give a general solution for AND, but cannot hope to give (general) transformations for the other two cases.

Below we consider combinations for arbitrary functions $f$ over a fixed ${ }^{2}$ number $q$ of predicates $\mathrm{P}_{1}, \mathrm{P}_{2}, \ldots, \mathrm{P}_{q}$. We assume that such a function $f\left(\mathrm{P}_{1}, \mathrm{P}_{2}, \ldots, \mathrm{P}_{q}\right)$ over the predicates itself constitutes a predicate and defines a set of derivable messages from $M$ in a straightforward way, by evaluating the predicates for $\left(M, m^{\prime}\right)$ and plugging the results into the formula. If viewed as sets, our basic examples for OR, AND, and NOT can then be written as $f_{\vee}\left(\mathrm{P}_{1}, \mathrm{P}_{2}\right)(M)=\mathrm{P}_{1}(M) \cup \mathrm{P}_{2}(M)$, and $f_{\wedge}\left(\mathrm{P}_{1}, \mathrm{P}_{2}\right)(M)=\mathrm{P}_{1}(M) \cap \mathrm{P}_{2}(M)$, as well as $f_{\neg}\left(\mathrm{P}_{1}\right)(M)=\mathcal{M} \backslash \mathrm{P}_{1}(M)$.

Note that one could more generally also define $f\left(\mathrm{P}_{1}, \mathrm{P}_{2}, \ldots, \mathrm{P}_{q}\right)$ for divisible message sets $M=$ $\left(M_{1}, M_{2}, \ldots, M_{q}\right)$ by evaluating $f\left(M, m^{\prime}\right)$ as a logical formula over $\mathrm{P}_{1}\left(M_{1}, m^{\prime}\right), \ldots, \mathrm{P}_{q}\left(M_{q}, m^{\prime}\right)$, i.e., assigning only the $i$-th part $M_{i}$ of $M$ to the $i$-th predicate, instead of using the same set $M$ for all predicates. This can be captured in our notion with a single $M$ by having the predicates $\mathrm{P}_{i}$ first project $M$ onto $M_{i}$ and then evaluating the actual predicate on $\left(M_{i}, m^{\prime}\right)$. For sake of readability we use the simpler notion with identical $M$.

We also assume that the message spaces $\mathcal{M}_{i}$ of all schemes are identical. This can always be achieved by setting $\mathcal{M}=\bigcap_{i=1}^{q} \mathcal{M}_{i}$. Note that, if message spaces are not identical this in principle allows to distinguish, say, in case of OR which predicate can be used to create a signature for some message. Since this would violate the idea of privacy immediately, we restrict ourselves to the case of identical message spaces.

\footnotetext{
${ }^{2}$ Note that, in general, the number of combined predicates is specific for the scheme and must not depend on the security parameter, i.e., the design of the scheme does not change with the security parameter. In this sense the number $q$ of predicates is constant in the security parameter.
} 


\subsection{Statically Adjustable Computations for AND}

We first confirm that the solution to sign each message component-wise under a set of public keys yields a secure solution for the AND. Instead of considering only two predicates we allow to combine any fixed number $q$ of predicates.

Construction 3.1 (AND-Construction) Let $\quad\left(\right.$ KeyGen $_{i}$, Sign $_{i}$, SignDer $_{i}$, Verify $\left._{i}\right) \quad$ be $\quad P_{i^{-}}$ homomorphic signature schemes for predicates $P_{1}, \ldots, P_{q}$. Then the following scheme (KeyGen, Sign, SignDer, Verify) is a $P$-homomorphic signature scheme for $P=P_{1} \wedge \ldots \wedge P_{q}$ :

- KeyGen $\left(1^{\lambda}\right)$ runs $\left(s k_{i}, p k_{i}\right) \leftarrow \operatorname{KeyGen}_{i}\left(1^{\lambda}\right)$ for all $i=1,2, \ldots, q$, and outputs $s k=$ $\left(s k_{1}, \ldots, s k_{q}\right)$ and $p k=\left(p k_{1}, \ldots, p k_{q}\right)$.

- $\operatorname{Sign}(s k, m, P)$ computes $\sigma_{i} \leftarrow \operatorname{Sign}_{i}\left(s k_{i}, m, P_{i}\right)$ for all $i$ and returns $\sigma=\left(\sigma_{1}, \ldots, \sigma_{q}\right)$.

- SignDer $\left(p k, M, \Sigma, m^{\prime}, P\right)$ first checks that $P_{i}\left(M, m^{\prime}\right)=1$ for all $i$, and then creates $\sigma_{i}^{\prime} \leftarrow$ $\operatorname{SignDer}_{i}\left(p k_{i}, M, \Sigma_{i}, m^{\prime}, P_{i}\right)$ where $\Sigma_{i}$ is the set of projections on the $i$-th component for each signature tuple in $\Sigma=\left\{\sigma_{m}\right\}_{m \in M}$. It returns $\sigma^{\prime}=\left(\sigma_{1}^{\prime}, \ldots, \sigma_{q}^{\prime}\right)$.

- Verify $(p k, M, \Sigma, P)$ returns 1 if and only if $\operatorname{Verify}_{i}\left(p k_{i}, M, \Sigma_{i}, P_{i}\right)=1$ for all $i$ (where again $\Sigma_{i}$ is the set of projections on the $i$-th component for each signature in $\Sigma$ ).

Correctness follows easily from the correctness of the underlying $\mathrm{P}_{i}$-homomorphic schemes.

Proposition 3.2 For any constant $q$ and any unforgeable and completely (resp. adaptively) contexthiding $P_{i}$-homomorphic schemes, Construction 3.1 (AND-Construction) is unforgeable and completely (resp. adaptively) context-hiding.

For concrete parameters our proof shows that the advantage of breaking unforgeability resp. context hiding for the AND scheme is bounded by the sum of the advantages for the corresponding property over all $\mathrm{P}_{i}$-homomorphic schemes.

Proof. We first show unforgeability, then context hiding.

Unforgeability. Assume that there exists a successful adversary $\mathcal{A}$ against unforgeability (Definition 2.2 for the $\mathrm{P}$-homomorphic signature scheme where $\mathrm{P}=\mathrm{P}_{1} \wedge \ldots \wedge \mathrm{P}_{q}$. For each $i \in\{1,2, \ldots, q\}$, we first construct an adversary $\mathcal{A}_{i}$ against the unforgeability of the underlying $\mathrm{P}_{i}$-homomorphic signature schemes:

- $\mathcal{A}_{i}$ initially receives $p k_{i}$ from the challenger $\mathcal{C}_{i}$ for the game against the $\mathrm{P}_{i}$-homomorphic signature schemes.

- $\mathcal{A}_{i}$ creates an initially empty table $T^{\prime}$ and runs $\left(s k_{j}, p k_{j}\right) \leftarrow \operatorname{KeyGen}_{j}\left(1^{\lambda}\right)$ for all $j=1,2, \ldots, q$, $j \neq i$ to create the other keys.

- $\mathcal{A}_{i}$ invokes adversary $\mathcal{A}$ against the AND-scheme on $p k=\left(p k_{1}, \ldots, p k_{q}\right)$.

- For every signing query $(m, \mathrm{P})$ from $\mathcal{A}$, adversary $\mathcal{A}_{i}$ creates a signing query for message $m$ and the predicate $\mathrm{P}_{i}$ for its challenger and gets the handle $h$, then computes $\sigma_{j} \leftarrow \operatorname{Sign}_{j}\left(s k_{j}, m, \mathrm{P}_{j}\right)$ for all $j \neq i$, and stores $\left(j, h, m, \sigma_{j}, \mathrm{P}_{j}\right)$ in $T^{\prime}$. 
- For every derivation query $\left(\{h\}, m^{\prime}, \mathrm{P}\right)$ of $\mathcal{A}$, adversary $\mathcal{A}_{i}$ passes a derivation query for the corresponding handles $\left(\{h\}, m^{\prime}, \mathrm{P}_{i}\right)$ to its challenger to receive a handle $h^{\prime}$. If $h^{\prime} \neq \perp$ adversary $\mathcal{A}_{i}$ looks up all entries $\left(j, h, m, \sigma_{m}, \mathrm{P}_{j}\right)$ for $j \neq i$ in $T^{\prime}$ for the queried handles in $\{h\}$ to form $M=\{m\}$, internally checks $\mathrm{P}_{j}\left(M, m^{\prime}\right)=1$, and computes $\sigma_{j}^{\prime} \leftarrow \operatorname{SignDer}_{j}\left(p k_{j}, M,\left\{\sigma_{m}\right\}_{m \in M}, m^{\prime}, \mathrm{P}_{j}\right)$. If no error occurs it returns $h^{\prime}$ to $\mathcal{A}$ and stores $\left(j, h^{\prime}, m^{\prime}, \sigma_{j}^{\prime}, \mathrm{P}_{j}\right)$ in $T^{\prime}$ for all $j \neq i$; else it returns $\perp$.

- For every reveal request $\mathcal{A}_{i}$ runs a reveal request for the corresponding handle $h$, combines the reply $\sigma_{i}$ with the values $\sigma_{j}$ from entries $\left(j, h, m, \sigma_{j}, \mathrm{P}_{j}\right)$ in $T^{\prime}$ to $\sigma$ and sends it to $\mathcal{A}$; in case of an error it simply returns $\perp$.

- When $\mathcal{A}$ eventually outputs a tuple $(m, \sigma, \mathrm{P})$, then $\mathcal{A}_{i}$ outputs the tuple $\left(m, \sigma_{i}, \mathrm{P}_{i}\right)$ for the $i$-th component $\sigma_{i}$ in $\sigma$.

Note that for each $i$ adversary $\mathcal{A}_{i}$ perfectly simulates an attack of $\mathcal{A}$ on the P-homomorphic scheme with the help of its challenger, such that $\mathcal{A}$ would output a successful forgery with the same probability in the simulation as in the original attack. By construction, we also have that the message set $M_{\mathrm{P}}$ of queries $(m, *, \mathrm{P})$ in $\mathcal{A}$ 's queries in the simulation is identical to the set $M_{\mathrm{P}_{i}}$ for queries $\left(m, *, \mathrm{P}_{i}\right)$ of $\mathcal{A}_{i}$ to its challenger for each $i$. Hence, from $m \notin \mathrm{P}^{*}\left(M_{\mathrm{P}}\right)$ it follows that $m \notin \mathrm{P}_{i}^{*}\left(M_{\mathrm{P}_{i}}\right)$ for some $i \in\{1,2 \ldots, q\}$. Furthermore, since verification succeeds for all components, it also holds that $\operatorname{Verify}_{i}\left(p k_{i}, m, \sigma, \mathrm{P}_{i}\right)=1$ for this $i$.

In other words, any successful forgery yields a successful forgery against (at least) one of the underlying schemes. It follows that the probability of breaking unforgeability for the AND scheme is bounded from above by the sum of the probabilities to break each underlying scheme.

Context Hiding. Assume next that there exists a successful adversary $\mathcal{A}$ against context hiding (Definition 2.3) for our $\mathrm{P}$-homomorphic signature scheme with $\mathrm{P}=\mathrm{P}_{1} \wedge \ldots \wedge \mathrm{P}_{q}$. As in the case of unforgeability we construct, for each $i \in\{1,2, \ldots, q\}$, an adversary $\mathcal{A}_{i}$ against context hiding of the $i$-th scheme. The advantage of $\mathcal{A}$ will be bounded from above by the sum over all advantages of the $\mathcal{A}_{i}$ 's via a standard hybrid argument. Furthermore, each $\mathcal{A}_{i}$ will be efficient if $\mathcal{A}$ is, such that the claim remains true for adaptive context hiding.

Adversary $\mathcal{A}_{i}$ receives a pair $\left(s k_{i}, p k_{i}\right)$ from its challenger and creates the other key pairs $\left(s k_{j}, p k_{j}\right)$ for $j \neq i$ by running $\operatorname{KeyGen}_{j}\left(1^{\lambda}\right)$. It hands $s k=\left(s k_{1}, \ldots, s k_{q}\right)$ and $p k=\left(p k_{1}, \ldots, p k_{q}\right)$ to adversary $\mathcal{A}$ and waits for the adversary to create a challenge request $M, \Sigma, m^{\prime}$. For each signature $\sigma_{m}$ in $\Sigma$ adversary $\mathcal{A}_{i}$ extracts the $i$-th component and thereby forms the set $\Sigma_{i}$. It passes $M, m^{\prime}$, and $\Sigma_{i}$ to its own challenger to receive a signature $\sigma_{i}^{\prime}$ (or an error message). It creates the signatures $\sigma_{j}^{\prime}$ for $j<i$ by running the signing algorithm on $m^{\prime}$; for $j>i$ it runs the signature derivation algorithm on $M, m^{\prime}, \Sigma_{j}$ to create the remaining signatures $\sigma_{j}^{\prime}$. In all cases it checks the validity of the predicates and signatures. If there is an error it returns $\perp$ to the adversary $\mathcal{A}$, and $\left(\sigma_{1}^{\prime}, \ldots, \sigma_{q}^{\prime}\right)$ otherwise. If $\mathcal{A}$ eventually outputs a bit $b^{*}$ then $\mathcal{A}_{i}$, too, outputs this bit and stops.

For the analysis note that $\mathcal{A}_{1}$, given that its challenger uses $b=0$, describes the case that all signatures are derived via SignDer. It follows that the probability of $\mathcal{A}$ correctly outputting 0 for derived signatures in the attack (and thus in the perfect simulation through $\mathcal{A}_{1}$ ) is exactly the probability that $\mathcal{A}_{1}$ returns 0 , given $b=0$ in its challenge. Analogously, given $b=1$ adversary $\mathcal{A}_{q}$ only creates fresh signatures via Sign in all components, hence given $b=1$ the probability that $\mathcal{A}_{q}$ returns 0 is exactly the same that $\mathcal{A}$ outputs 0 in the case that all signatures are fresh. A standard 
hybrid argument now yields:

$$
\begin{aligned}
\operatorname{Prob}[\mathcal{A}=b]-\frac{1}{2}=\frac{1}{2} \cdot(\operatorname{Prob}[\mathcal{A}=0 \mid b=0]-\operatorname{Prob}[\mathcal{A}=0 \mid b=1]) \\
=\frac{1}{2} \cdot\left(\operatorname{Prob}\left[\mathcal{A}_{1}=0 \mid b=0\right]-\operatorname{Prob}\left[\mathcal{A}_{q}=0 \mid b=1\right]\right) \\
=\frac{1}{2} \cdot\left(\operatorname{Prob}\left[\mathcal{A}_{1}=0 \mid b=0\right]-\operatorname{Prob}\left[\mathcal{A}_{q}=0 \mid b=1\right]\right. \\
\left.\quad+\sum_{i=1}^{q-1}\left(\operatorname{Prob}\left[\mathcal{A}_{i}=0 \mid b=1\right]-\operatorname{Prob}\left[\mathcal{A}_{i}=0 \mid b=1\right]\right)\right)
\end{aligned}
$$

and observing that $\operatorname{Prob}\left[\mathcal{A}_{i}=a \mid b=1\right]=\operatorname{Prob}\left[\mathcal{A}_{i+1}=a \mid b=0\right]$, because in both cases exactly the first $i$ signatures are computed via Sign,

$$
\begin{aligned}
= & \frac{1}{2} \cdot\left(\operatorname{Prob}\left[\mathcal{A}_{1}=0 \mid b=0\right]-\operatorname{Prob}\left[\mathcal{A}_{q}=0 \mid b=1\right]\right. \\
& \left.\quad+\sum_{i=1}^{q-1}\left(\operatorname{Prob}\left[\mathcal{A}_{i+1}=0 \mid b=0\right]-\operatorname{Prob}\left[\mathcal{A}_{i}=0 \mid b=1\right]\right)\right) \\
= & \sum_{i=1}^{q} \frac{1}{2} \cdot\left(\operatorname{Prob}\left[\mathcal{A}_{i}=0 \mid b=0\right]-\operatorname{Prob}\left[\mathcal{A}_{i}=0 \mid b=1\right]\right) \\
= & \sum_{i=1}^{q} \operatorname{Adv}\left(\mathcal{A}_{i}\right) .
\end{aligned}
$$

This proves context hiding.

\subsection{Statically Adjustable Computations for OR and NOT}

Our impossibility result holds for canonical constructions which combine $\mathrm{P}_{i}$-homomorphic schemes in a general way, ruling out specific constructions which ignore the underlying schemes and builds a new scheme from scratch. We require four algorithms, one for synthesizing public keys of the individual schemes into one for the combined scheme (PKComb), one for splitting keys (PKSplit), one for combining signatures (SigComb), and one to divide signatures for the combined scheme into signatures for the individual schemes (SigSplit). The latter is usually necessary to reduce the security to the security of the individual schemes.

For sake of readability we follow the statistical indistinguishability approach also used for (complete) context hiding, and require that the distributions of the algorithms above for combining and splitting keys and signatures have identical distributions as if running the actual algorithms of the combined scheme directly. As our proof below shows our impossibility result can be extended to cover computationally indistinguishable distributions.

Definition 3.3 (Canonical Construction) Let $f$ be a functional predicate over predicates $P_{1}, \ldots, P_{q}$ for a fixed number $q$ of predicates. A statically adjustable $f\left(P_{1}, \ldots, P_{q}\right)$ homomorphic signature scheme (KeyGen, Sign, SignDer, Verify) is a canonical construction out of $P_{i}$-homomorphic signature schemes $\left(K_{\text {eyGen }}\right.$, Sign $_{i}$, SignDer $_{i}$, Verify $\left._{i}\right)$ if there exist PPT algorithms (PKComb, PKSplit, SigComb, SigSplit) such that:

Identical distribution of combined keys: The following random variables are identically distributed:

$$
\text { - Let }(p k, s k) \leftarrow \operatorname{KeyGen}\left(1^{\lambda}\right) \text { and output } p k \text {; }
$$


- Let $\left(p k_{i}, s k_{i}\right) \leftarrow \operatorname{KeyGen}_{i}\left(1^{\lambda}\right)$ for all $i, p k \leftarrow P K \operatorname{Comb}\left(p k_{1}, \ldots, p k_{q}\right)$, output $p k$,

Identical distribution of split keys: The following random variables are identically distributed:

- Let $(p k, s k) \leftarrow \operatorname{KeyGen}\left(1^{\lambda}\right)$ and output $\left(p k_{1}, \ldots, p k_{q}\right) \leftarrow P K S p l i t(p k)$;

- Let $\left(p k_{i}, s k_{i}\right) \leftarrow \operatorname{KeyGen}_{i}\left(1^{\lambda}\right)$ for all $i$, output $\left(p k_{1}, \ldots, p k_{q}\right)$,

Identical distribution of combined signatures: For any PPT algorithm $\mathcal{F}$ the following pairs of random variables are identically distributed:

- Run $M \leftarrow \mathcal{F}\left(1^{\lambda}\right) . \quad$ Compute $(p k, s k) \leftarrow$ KeyGen $\left(1^{\lambda}\right)$ and output $\Sigma \leftarrow \operatorname{Sign}\left(s k, M, f\left(P_{1}, \ldots, P_{q}\right)\right) ;$

- Run $M \leftarrow \mathcal{F}\left(1^{\lambda}\right)$. For all $i$, compute $\left(p k_{i}, s k_{i}\right) \leftarrow \operatorname{KeyGen}_{i}\left(1^{\lambda}\right)$ along with $\Sigma_{i} \leftarrow$ $\operatorname{Sign}_{i}\left(s_{i}, M, P_{i}\right)$. Synthesize the public key via $p k \leftarrow P K \operatorname{Comb}\left(p k_{1}, \ldots, p k_{q}\right)$ and output $\Sigma \leftarrow \operatorname{Sig} \operatorname{Comb}\left(p k, p k_{1}, \ldots, p k_{q}, \Sigma_{1}, \ldots, \Sigma_{q}, M\right)$.

Splitting Signatures: For any PPT algorithm $\mathcal{F}^{\prime}$ we have that for $\left(p k_{i}, s k_{i}\right) \leftarrow$ $\operatorname{KeyGen}_{i}\left(1^{\lambda}\right)$ for all $i, \quad p k \leftarrow \operatorname{PKComb}\left(p k_{1}, \ldots, p k_{q}\right), \quad\left(M, m^{\prime}\right) \leftarrow \mathcal{F}^{\prime}\left(1^{\lambda}\right)$ where $m^{\prime} \in f\left(P_{1}, \ldots, P_{q}\right)(M), \Sigma_{i} \leftarrow \operatorname{Sign}_{i}\left(s k_{i}, M, P_{i}\right), \Sigma \leftarrow \operatorname{SigComb}\left(p k_{1}, \ldots, p k_{q}, \Sigma_{1}, \ldots, \Sigma_{q}, M\right)$, $\sigma^{\prime} \leftarrow \operatorname{SignDer}\left(p k, M, \Sigma, m^{\prime}, f\left(P_{1}, \ldots, P_{q}\right)\right)$, the probability that $\left(\sigma_{1}^{\prime}, \ldots, \sigma_{q}^{\prime}\right) \leftarrow$ SigSplit $\left(p k, p k_{1}, \ldots, p k_{q}, m^{\prime}, \sigma^{\prime}\right)$ does not contain some valid component and thus Verify $_{i}\left(p k_{i}, m^{\prime}, \sigma_{i}^{\prime}\right)=0$ for all $i$, is negligible.

In other words, SigSplit returns at least one valid signature for one of the underlying predicates with sufficiently high probability. Our AND-construction is canonical in the above sense: PKComb and SigComb both concatenate their inputs (and PKSplit divides the concatenated keys again), and SigSplit simply returns the signature itself. Note that the definition allows PKComb, PKSplit, SigComb, and SigSplit to depend on the given predicates $\mathrm{P}_{i}$; the construction only follows a canonical pattern.

In what follows, we need to exclude trivial examples like $P_{1} \vee P_{2}=P_{1} \vee P_{1}$. Hence, for the $O R$ we assume below the existence of a message $m^{\prime}$ which can be derived from a set of messages $M$ under one predicate, but not the other predicate. This clearly prevents $\mathrm{P}_{1}=\mathrm{P}_{2}$. More generally, and to include for instance also the NOT case, we assume that $m^{\prime}$ can be derived under $f\left(\mathrm{P}_{1}, \mathrm{P}_{2}, \ldots\right)$ but not under one of the predicates; the excluded predicate $\mathrm{P}_{i}$ can be arbitrary, but the output distribution of $m^{\prime}$ does not depend on this choice. The latter is necessary to ensure that $m^{\prime}$ does not contain any information about the predicate's index $i$. Furthermore, we assume that such pairs $\left(M, m^{\prime}\right)$ are efficiently computable. We discuss an illuminating example after the definition.

Definition 3.4 (Efficiently Distinguishable Predicates) Let $f$ be a functional predicate over predicates $P_{1}, \ldots, P_{q}$. Consider a statically adjustable $f\left(P_{1}, \ldots, P_{q}\right)$-homomorphic signature scheme (KeyGen, Sign, SignDer, Verify). Then the predicates are called efficiently distinguishable with respect to $f$, if there exists a PPT algorithm $\mathcal{F}$ such that for any $i \in\{1,2, \ldots, q\}$ and for any $\left(M, m^{\prime}\right) \leftarrow \mathcal{F}\left(1^{\lambda}, i\right)$, we have $m^{\prime} \in\left(f\left(P_{1}, \ldots, P_{q}\right)(M) \backslash P_{i}^{*}(M)\right)$. Moreover, for any $i, j \in$ $\{1,2, \ldots, q\}$ the distribution of $m^{\prime}$ (over the coin tosses of $\mathcal{F}$ ) in the output of $\mathcal{F}\left(1^{\lambda}, i\right)$ resp. $\mathcal{F}\left(1^{\lambda}, j\right.$ ) is identical.

Let us demonstrate the property for the introductory example of two redactable signature schemes (with message space $\mathcal{M}=\{0,1\}^{*}$ ), one allowing to drop message bits only at the front (predicate $\mathrm{P}_{1}$ ), and the other one only at the end $\left(\mathrm{P}_{2}\right)$. Consider the OR predicate $\mathrm{P}_{1} \vee \mathrm{P}_{2}$ describing a scheme for quotable substrings. Then $\mathcal{F}$ can simply pick $m^{\prime}=0^{\lambda}$ and for $i=1$ output $M=\left\{0^{\lambda} 1\right\}$, 
and for $i=2$ it returns $M=\left\{10^{\lambda}\right\}$ instead. Clearly, for $i=1$ one can derive $m^{\prime}$ from $M$ via $\mathrm{P}_{2}$ and therefore for the OR, but not via $\mathrm{P}_{1}$, because the ' 1 ' at the end cannot be redacted through $\mathrm{P}_{1}$. The same argument holds vice versa for $i=2$, and the (trivial) distributions on $m^{\prime}$ are identical for both $i=1$ and $i=2$. Hence, this examples has efficiently distinguishable predicates.

The case of NOT is even simpler. Algorithm $\mathcal{F}$ simply needs to find some $M$ and some $m^{\prime}$ which lies in $(\neg \mathrm{P}(M)) \backslash \mathrm{P}^{*}(M)=\mathcal{M} \backslash \mathrm{P}^{*}(M)$, i.e., if $m^{\prime}$ is not derivable according to $\mathrm{P}^{*}(M)$. Finally note that constructions based only on AND cannot be distinguishable, since $\left(\mathrm{P}_{1}(M) \cap \mathrm{P}_{2}(M)\right) \backslash \mathrm{P}_{i}^{*}(M)=$ $\emptyset$ for any $i$.

Theorem 3.5 Let $f$ be a functional predicate over predicates $P_{1}, \ldots, P_{q}$ for a fixed number $q$ of predicates. Assume further that the predicates are efficiently distinguishable with respect to $f$. Then there is no adaptively context-hiding, statically-adjustable $f\left(P_{1}, \ldots, P_{q}\right)$-homomorphic signature scheme which is a canonical construction out of unforgeable $P_{i}$-homomorphic signature schemes.

The proof idea is as follows. Essentially we show how to forge a signature for one of the underlying schemes. For this we use the distinguishability of the predicates to create a set of messages $M$ and a message $m^{\prime}$ which is derivable by $f\left(\mathrm{P}_{1}, \ldots, \mathrm{P}_{q}\right)(M)$ but does not lie in $\mathrm{P}_{i}^{*}(M)$ for some $i$. Then we ask for signatures for the messages in $M$, and derive a signature for $m^{\prime}$ via the public operation SignDer for the combined scheme and for $f\left(\mathrm{P}_{1}, \ldots, \mathrm{P}_{q}\right)$. Splitting up the signature into its components via SigSplit we obtain (with sufficiently large probability) a valid signature for $m^{\prime}$ under the $i$-th scheme. But since $m^{\prime} \notin \mathrm{P}_{i}^{*}(M)$ we thus create a valid forgery, contradicting the security of the underlying scheme. In the course of the proof we use the context hiding property to show that the "skewed" choice of $M, m^{\prime}$ (with $m^{\prime} \notin \mathrm{P}_{i}^{*}(M)$ ) does not bias the success probability of SigSplit for returning a valid signature component for the $i$-th scheme significantly.

Proof. Take an arbitrary canonical construction. By the efficient distinguishability of the predicates there exists a PPT algorithm $\mathcal{F}$ which on input $i$ returns $\left(M, m^{\prime}\right)$, such that $m^{\prime} \in$ $\left(f\left(\mathrm{P}_{1}, \ldots, \mathrm{P}_{q}\right) \backslash \mathrm{P}_{i}^{*}\right)(M)$. Given this algorithm we next construct, for each $i \in\{1,2, \ldots, q\}$, an adversary $\mathcal{A}_{i}$ trying to break the unforgeability game of the $\mathrm{P}_{i}$-homomorphic signature scheme $\left(\right.$ KeyGen $_{i}, \operatorname{Sign}_{i}, \operatorname{SignDer}_{i}$, Verify $\left._{i}\right)$ :

- The adversary $\mathcal{A}_{i}$ is given $p k_{i}$ of $\left(s k_{i}, p k_{i}\right) \leftarrow \operatorname{KeyGen}_{i}\left(1^{\lambda}\right)$. It generates the other keys $\left(p k_{j}, s k_{j}\right) \leftarrow \operatorname{KeyGen}_{j}\left(1^{\lambda}\right)$ for $j \neq i$ on its own. It also computes the combined public key $p k \leftarrow \operatorname{PKComb}\left(p k_{1}, \ldots, p k_{q}\right)$.

- $\mathcal{A}_{i}$ runs $\mathcal{F}$ on $\left(1^{\lambda}, i\right)$ to find a suitable tuple $\left(M, m^{\prime}\right)$.

- It obtains signatures for the set $M$ with the help of signing queries, namely $\Sigma_{i} \leftarrow$ $\operatorname{Sign}_{i}\left(s k_{i}, M, \mathrm{P}_{i}\right)$. It computes the other signatures $\Sigma_{j} \leftarrow \operatorname{Sign}_{j}\left(s k_{j}, M, \mathrm{P}_{j}\right)$ with the secret keys $s k_{j}$, and synthesizes them all via $\Sigma \leftarrow \operatorname{Sig} \operatorname{Comb}\left(p k, p k_{1}, \ldots, p k_{q}, \Sigma_{1}, \ldots, \Sigma_{q}, M\right)$.

- Adversary $\mathcal{A}_{i}$ obtains the forged signature by the derivative $\sigma^{\prime} \leftarrow$ $\operatorname{Sign\operatorname {Der}}\left(p k, M, \Sigma, m^{\prime}, f\left(\mathrm{P}_{1}, \ldots, \mathrm{P}_{q}\right)\right) \quad$ and $\quad$ by running $\left(\sigma_{1}^{\prime}, \ldots, \sigma_{q}^{\prime}\right) \quad \leftarrow$ $\operatorname{SigSplit}\left(p k, p k_{1}, \ldots, p k_{q}, m^{\prime}, \sigma^{\prime}\right)$. It returns $\sigma_{i}^{\prime}$.

Obviously, adversary $\mathcal{A}_{i}$ runs in polynomial time because all steps can be executed efficiently and the set $M$ is of at most polynomial size and therefore the number of signature queries and creations.

It remains to argue that the adversary outputs a valid forgery with non-negligible probability. To this end we need to show that the splitting algorithm returns with a valid signature for our 
$\mathrm{P}_{i}$-homomorphic scheme with sufficiently large probability. In particular, since $M, m^{\prime}$ are such that $m^{\prime} \in\left(f\left(\mathrm{P}_{1}, \ldots, \mathrm{P}_{q}\right) \backslash \mathrm{P}_{i}^{*}\right)(M)$ it may be that the signatures for $M$ and the input $m^{\prime}$ for SigSplit convey some information about $i$, and SigSplit then "avoids" to output some valid signature for $i$, but always uses a different index $j$. In fact, we need to make sure that SigSplit for our specific input does not return a valid signature for the $i$-th scheme with negligible probability, despite succeeding with non-negligible probability in general. We use the context hiding property to show that this cannot happen.

By assumption, the canonical construction is adaptively context hiding. We claim that the probability that SigSplit does not return a valid signature for the index $i$ given to $\mathcal{F}$ is negligible. To this end assume that SigSplit, instead of receiving the derived signature $\sigma^{\prime}$, is run on a fresh signature $\sigma^{\prime}$ generated through Sig on $m^{\prime}$ for a key $s k$ which is generated according to the key generation for the canonical construction, $(s k, p k) \leftarrow \operatorname{KeyGen}\left(1^{\lambda}\right)$. We claim that this cannot increase the probability of SigSplit returning a valid signature for the index $i$ given to $\mathcal{F}$ by more than a negligible amount. Otherwise it we can devise a successful (and efficient!) adversary $\mathcal{B}_{i}$ against the context hiding property, as explained next.

The adversary $\mathcal{B}_{i}$ would receive a key pair $(s k, p k) \leftarrow \operatorname{KeyGen}\left(1^{\lambda}\right)$ of the canonical construction, and mimic $\mathcal{A}_{i}$ 's behavior by running $\mathcal{F}$ on $i$ to receive $\left(M, m^{\prime}\right)$. It creates all signatures $\Sigma$ for the messages in $M$ with the secret key $s k$, and then calls its challenge oracle about $\Sigma, M, m^{\prime}$ to obtain a signature $\sigma^{\prime}$, either derived via SignDer or via Sign. It then runs SigSplit on $\left(p k, p k_{1}, \ldots, p k_{q}, m^{\prime}, \sigma^{\prime}\right)$ to obtain signatures for the individual schemes, where it derives the keys $p k_{1}, \ldots, p k_{q}$ via the key splitting algorithm PKSplit. It returns 0 if this yields a valid signature $\sigma_{i}^{\prime}$ for the index $i$, which it can decide by running the verification algorithm Verify ${ }_{i}$ on $\left(p k_{i}, m^{\prime}, \sigma_{i}^{\prime}, \mathrm{P}_{i}\right)$; else it returns 1.

Note that from SigSplit's perspective the simulations through $\mathcal{A}_{i}$ and through $\mathcal{B}_{i}$ (given $b=0$ and thus a derived signature via SignDer) are identical, because the distribution of combined keys and signatures and genuine keys are identical. The same is true for $\mathcal{A}_{i}$ 's run if giving SigSplit a fresh signature for $m^{\prime}$ instead, and $\mathcal{B}_{i}$ 's simulation for $b=1$. Hence, if the probability in $\mathcal{A}_{i}$ 's simulation, when giving a fresh signature for $m^{\prime}$ instead, to find a suitable signature for the $i$ th scheme would increase from negligible to non-negligible, then this would also be true for the two cases in $\mathcal{B}_{i}$ 's attack, contradicting the context hiding property. Hence, since $m^{\prime}$ is distributed independently of $i$ according to the efficient distinguishability requirement on $\mathcal{F}$, the index $i$ is information-theoretically hidden from algorithm SigSplit when receiving $p k, p k_{1}, \ldots, p k_{q}, \sigma^{\prime}, m^{\prime}$ for a fresh signature $\sigma^{\prime}$. In this case, the output by SigSplit does not depend on $i$, and by context hiding this remains true up to a negligible error for the "skewed" parameters $M, m^{\prime}$ and the derived signature via SignDer as chosen by $\mathcal{A}_{i}$ (for any $i$ ).

Now we can complete the argument of SigSplit outputting a valid signature for some $i$ sufficiently often. Recall that, for infinitely many security parameters $\lambda$, algorithm SigSplit returns some valid signature with a success probability exceeding a polynomial fraction in the sense of the definition of a canonical construction. Hence, among the (constant number of) $q$ predicates there must be a fixed index $i \in\{1,2, \ldots, q\}$ such that infinitely often the success probability exceeds a polynomial fraction for this index $i$. With the argument above, up to a negligible error, this remains true when run by $\mathcal{A}_{i}$ for the parameters $M, m^{\prime}$ which depend on $i$ now. Hence, this adversary still successfully forges infinitely often with sufficiently high probability. This, however, would contradict unforgeability of this scheme, concluding the proof.

We stress that the impossibility result holds for the computational notion of adaptive context hiding (with efficient distinguishers), which even strengthens our result. As mentioned before, a slightly more involved argument allows to extend the result also to algorithms PKComb, PKSplit, SigComb whose output is only computationally indistinguishable from the one 
of the original algorithms (instead of being identical). This requires some additional steps to prove that gradually replacing the algorithms does not change the behavior of SigSplit in the above proof significantly.

\section{Dynamically Adjustable Computations}

In the dynamic case we assume a polynomial universe $\mathcal{P}$ of predicates such that there exists a $\mathrm{P}_{i}$-homomorphic scheme for each $\mathrm{P}_{i} \in \mathcal{P}$. We furthermore assume that given (a description of) $\mathrm{P}_{i}$ one can efficiently recover the corresponding scheme, e.g., if the universe consists only of a fixed number of predicates. Vice versa, we assume that $\mathrm{P}_{i}$ is identifiable from the scheme's public key $p k_{i}$. This in particular implies that the public keys for predicates must be unique. For simplicity we assume an ordering on predicates in $\mathcal{P}$ and often identify the predicate $\mathrm{P}_{i}$ and the scheme with its number $i$ according to this order. We simply call sets $\mathcal{P}$ as above efficient.

In the construction we need to assume that for a given predicate identifier $i$ there is a fixed yet (pseudo)random key pair $\left(s k_{i}, p k_{i}\right) \leftarrow \operatorname{KeyGen}_{i}\left(1^{\lambda}\right)$, generated according to the key generation algorithm for the scheme for predicate $\mathrm{P}_{i}$. This key pair remains identical for all signature requests for $\mathrm{P}_{i}$. For a polynomial universe $\mathcal{P}$ this can be in principle implemented by generating the keys $\left(s k_{i}, p k_{i}\right)$ when creating the scheme's keys $(s k, p k)$, and storing them in $s k$. In practice this may indeed be admissible for a small number of predicates, a more applicable approach may be to generate the keys on the fly via a pseudorandom function. Namely, store a key $\kappa$ of a pseudorandom function in $s k$, and to create the key pair for predicate $\mathrm{P}_{i}$, recover the (pseudo)random output $\omega_{i}=\operatorname{PRF}\left(\kappa, \mathrm{P}_{i}\right)$ and re-run $\operatorname{KeyGen}_{i}\left(1^{\lambda} ; \omega_{i}\right)$ for $\omega_{i}$ to derive the same pair $\left(s k_{i}, p k_{i}\right)$ as before. For unforgeability it can be formally shown via standard techniques that this solution is (quasi) as secure as generating fresh key pairs and maintaining a table to look up previous keys; for context hiding, however, one requires an additional assumption on the security of the underlying scheme to preserve privacy, as discussed below.

Similarly, the public keys $p k_{i}$ and their (fixed) certificates cert $t_{i}$ may be published at once, or may be attached to each signature upon creation. Below we adopt the latter solution as it rather complies with our notion of (stateless) $\mathcal{P}$-homomorphic signatures. Hence, below we assume for simplicity that the efficient universe $\mathcal{P}$ stores all pairs $\left(s k_{i}, p k_{i}\right)$ with once-created certificates cert $_{i}$ at the beginning in $s k$. For certification we use a regular signature scheme which we can subsume as a special case under P-homomorphic schemes, without considering a SignDer algorithm nor context hiding. If we define $\mathrm{P}(M)=M$ for this scheme, unforgeability for this "homomorphic" scheme corresponds to the common notion of unforgeability for regular schemes.

Construction 4.1 (Certify-Then-Sign Construction) Let $\mathcal{P}$ be an efficient set of predicates $P_{1}, P_{2}, \ldots, P_{q}$. Let $\left.\left(K_{\text {eyGen }}, \text { Sign }_{0} \text {, Verify }\right)_{0}\right)$ be a regular signature scheme. Define the following dynamically adjustable $\mathcal{P}$-homomorphic signature scheme (KeyGen, Sign, SignDer, Verify):

- KeyGen $\left(1^{\lambda}\right)$ generates $\left(s k_{0}, p k_{0}\right) \leftarrow K_{\text {eyGen }}\left(1^{\lambda}\right)$, generates key pairs $\left(s k_{i}, p k_{i}\right) \leftarrow \operatorname{KeyGen}_{i}\left(1^{\lambda}\right)$ for all predicates $P_{i}$, and certificates cert $\leftarrow \operatorname{Sign}_{0}\left(s k_{0}, p k_{i}\right)$ for all $i$. It returns sk $=$ $\left(s k_{0},\left\{\left(s k_{i}, p k_{i}, \text { cert }_{i}\right)\right\}_{i}\right)$ and $p k=p k_{0}$.

- $\operatorname{Sign}\left(s k, m, P_{i}\right)$ looks up $\left(s k_{i}, p k_{i}\right.$, cert $\left._{i}\right)$ for $P_{i}$ in sk and computes $\sigma_{i} \leftarrow \operatorname{Sign}_{i}(s k, m)$ and returns $\sigma=\left(\sigma_{i}, p k_{i}\right.$, cert $\left._{i}\right)$.

- SignDer $\left(p k, M, \Sigma, m^{\prime}, P^{\prime}\right)$ checks that all signatures carry the same $k_{i}$ and cert for $_{\text {predicate }}$ $P_{i}$, that $P^{\prime}=P_{i}$, that $P_{i}\left(M, m^{\prime}\right)=1$, that Verify ${ }_{i}\left(p k_{i}, M, \Sigma\right)=1$, and, if all checks succeed, computes $\sigma_{i}^{\prime} \leftarrow \operatorname{SignDer}_{i}\left(p k_{i}, M, \Sigma, m^{\prime}\right)$ and returns $\sigma^{\prime}=\left(\sigma_{i}^{\prime}\right.$, pk $_{i}$, cert $\left._{i}\right)$. 
- Verify $(p k, m, \sigma, P)$ checks that $P$ corresponds to the public key in $\left(\sigma_{i}, p k_{i}\right.$, cert $\left._{i}\right)$, that $\operatorname{Verify}_{0}\left(p k, p k_{i}\right.$, cert $\left._{i}\right)=1$, and that $\operatorname{Verify}_{i}\left(p k_{i}, m, \sigma_{i}\right)=1$. Only if all checks succeed, it returns 1.

It is straightforward to verify that the above construction is correct in the sense that genuine (fresh and derived) signatures are accepted by Verify. This follows from the correctness properties of the regular scheme and of the $\mathrm{P}_{i}$-homomorphic ones.

Proposition 4.2 Assume that the signature scheme $\left(\mathrm{KeyGen}_{0}, \mathrm{Sign}_{0}\right.$, Verify $\left._{0}\right)$ and all $P_{i^{-}}$ homomorphic schemes are unforgeable according to Definition 2.2. Then the Certify-then-Sign Construction 4.1 is also unforgeable for the efficient universe $\mathcal{P}=\left\{P_{1}, \ldots, P_{q}\right\}$ for the fixed number $q$ of predicates.

In terms of concrete security, the success probability of any adversary against the construction is (for similar running time) bounded from above by the probability of forging certificates, plus $q$ times the maximal advantage against any of the schemes from $\mathcal{P}$.

Proof. Assume that there exists a successful forger $\mathcal{A}$. Then this adversary is able to forge with non-negligible probability a signature $\sigma^{*}=(\sigma, p k, c e r t)$ for a message $m$ such that, in particular, $\operatorname{Verify}_{0}\left(p k_{0}, p k, c e r t\right)=1$. Note that if the probability that $\mathcal{A}$ succeeds and that $p k$ does not match any of the keys $p k_{i}$ created by the signer for the predicates $\mathrm{P}_{i}$, was non-negligible, then this would straightforwardly contradict the unforgeability of the certification scheme. Namely, construct an algorithm $\mathcal{A}_{0}$ against the certification scheme which, on input $p k_{0}$, creates the polynomial number of key pairs $\left(s k_{i}, p k_{i}\right) \leftarrow \operatorname{KeyGen}_{i}\left(1^{n}\right)$ and asks for signatures cert $t_{i}$ for all $p k_{i}$ from the signing oracle, and then emulates the attack of $\mathcal{A}$ with the help of the secret keys. If $\mathcal{A}$ eventually outputs $\sigma^{*}=(\sigma, p k, c e r t)$, then $\mathcal{A}_{0}$ returns $p k$, cert as the forgery attempt.

If the probability that $\mathcal{A}$ would succeed for a fresh $p k$ with non-negligible probability as defined above, then our efficient algorithm $\mathcal{A}_{0}$, which perfectly simulates the actual attack, would then successfully forge a signature cert for a new "message" $p k$ with non-negligible probability. Since this would contradict the unforgeability of the certification scheme, we can assume that this case happens with negligible probability only. It follows that $\mathcal{A}$ must succeed with non-negligible probability for a key $p k=p k_{i}$ for some (unique) $i$, such that $\operatorname{Verify}_{i}\left(p k_{i}, m, \sigma, \mathrm{P}_{i}\right)=1$, and the message is not trivially derivable under the corresponding predicate $\mathrm{P}_{i}$ from the signing queries for $\mathrm{P}_{i}$.

Note that the specific choice $p k_{i}$ may depend on the adversary's randomness. However, there must exist at least one predicate $\mathrm{P}_{i}$ (among the $q$ schemes) such that $\mathcal{A}$ succeeds for this key fixed $p k_{i}$ with non-negligible probability. We can now derive an adversary $\mathcal{A}_{i}$ successfully forging signatures for this $\mathrm{P}_{i}$-homomorphic scheme. Adversary $\mathcal{A}_{i}$ receives from the challenger the public key $p k_{i}$ and gets access to a $\operatorname{Sign}_{i}$-oracle. It generates $\left(s k_{0}, p k_{0}\right)$ and all other key pairs $\left(s k_{j}, p k_{j}\right)$ and signs all of them, including $p k_{i}$. The adversary $\mathcal{A}_{i}$ then runs $\mathcal{A}$ on $p k_{0}$, supplying all signatures requests for $\mathrm{P}_{j} \neq \mathrm{P}_{i}$ with the help of the secret keys, and using the external signing oracle for $\mathrm{P}_{i}$. If $\mathcal{A}$ finally returns $m$ and $(\sigma, p k, c e r t)$ then $\mathcal{A}_{i}$ returns $m$ and $\sigma_{i}$.

Note that, if $\mathcal{A}$ has a non-negligible success probability for forging under the key $p k_{i}$, then $\mathcal{A}_{i}$ has the same success probability. This follows as the signature verifies under $p k_{i}$, and if the message $m$ is not derivable from $\mathcal{A}$ 's queries for $\mathrm{P}_{i}$, then this is also true for $\mathcal{A}_{i}$. This, however, would contradict the unforgeability assumption about the $\mathrm{P}_{i}$-homomorphic scheme.

Proposition 4.3 Assume that all $P_{i}$-homomorphic schemes are completely (resp. adaptively) context-hiding according to Definition 2.3. Then the Certify-then-Sign Construction 4.1 is also completely (resp. adaptively) context-hiding for an efficient universe $\mathcal{P}=\left\{P_{1}, P_{2}, \ldots, P_{q}\right\}$ of a fixed number $q$ of predicates. 
Proof. Assume that there exists a successful adversary $\mathcal{A}$ with non-negligible advantage. We will show that at least one predicate will not be completely (resp. adaptively) context-hiding. For any $i \in\{1, \ldots, q\}$ construct an adversary $\mathcal{A}_{i}$ that plays against the predicate $\mathrm{P}_{i}$. This adversary $\mathcal{A}_{i}$ receives from the challenger the private key and the public key $\left(s k_{i}, p k_{i}\right)$ for the $i$-th scheme. It then generates $\left(s k_{0}, p k_{0}\right) \leftarrow \operatorname{KeyGen}_{0}\left(1^{\lambda}\right)$, the key pairs $\left(s k_{j}, p k_{j}\right)$ for all $j \neq i$, and creates the certificates cert $_{i}$ for all $i$ according to our construction. The private key $s k=\left(s k_{0},\left\{\left(s k_{i}, p k_{i}, \text { cert }_{i}\right)\right\}_{i}\right)$ and the public key $p k=p k_{0}$ are then passed to adversary $\left.\mathcal{A}\right]^{3}$ Adversary $\mathcal{A}$ then selects $\left(M, \Sigma, m^{\prime}, \mathrm{P}_{j}\right)$. In the case of $i=j$ the adversary $\mathcal{A}_{i}$ forwards it to the challenger. The challenger then returns either a derived signature, or a fresh signature $\sigma^{\prime}$. The adversary $\mathcal{A}_{i}$ then returns the final output bit $b^{*}$ of $\mathcal{A}$. For the case $i \neq j$, adversary $\mathcal{A}_{i}$ simply returns a random bit $b^{*} \leftarrow\{0,1\}$.

Note that, if $\mathcal{A}$ has a non-negligible advantage for the combined scheme, this means that it also has a non-negligible advantage when conditioning on $\mathcal{A}$ choosing one of the fixed predicates $\mathrm{P}_{i}$ for the challenge (involving $\mathrm{P}_{j}$ ). More precisely, there must be some $i$ such that the probability of $\mathcal{A}$ predicting correctly the bit $b$ (in the actual attack and in the perfectly indistinguishable simulation through $\mathcal{A}_{i}$ ) and picking $j=i$, is at least $\frac{1}{q}$ times the general prediction probability. Since the number $q$ of predicates is fixed this probability must be non-negligible as well. Fix such an $i$ from now on.

The advantage of $\mathcal{A}_{i}$ (against the underlying scheme) in terms of the advantage of $\mathcal{A}$ (against the derived scheme) is then given by

$$
\begin{aligned}
\operatorname{Adv}\left(\mathcal{A}_{i}\right) & =\operatorname{Prob}\left[\mathcal{A}_{i}=b\right]-\frac{1}{2} \\
& =\operatorname{Prob}[\mathcal{A}=b \wedge i=j]+\operatorname{Prob}\left[b^{*}=b \wedge i \neq j\right]-\frac{1}{2} \\
& \geq \frac{1}{q} \cdot \operatorname{Prob}[\mathcal{A}=b]+\frac{1}{2} \cdot\left(1-\frac{1}{q}\right)-\frac{1}{2} \\
& \geq \frac{1}{q} \cdot \operatorname{Adv}(\mathcal{A})
\end{aligned}
$$

Hence, if $\mathcal{A}$ breaks context hiding, i.e., has some non-negligible success probability, at least one of the adversary $\mathcal{A}_{i}$ also breaks context hiding for $\mathrm{P}_{i}$ (losing a factor $\frac{1}{q}$ in the advantage compared to $\mathcal{A}$ ). This predicate would thus not be completely (resp. adaptively) context-hiding, which contradicts our assumption.

If we use a pseudorandom function with key $\kappa$ to create $\omega_{i}=\operatorname{PRF}\left(\kappa, \mathrm{P}_{i}\right)$, then to inherit the context hiding property we need to assume that giving $\omega_{i}$ (in addition to $s k_{i}$ ) to the distinguisher does not violate context hiding of the underlying $\mathrm{P}_{i}$-homomorphic scheme. If this is the case then we can formally reduce security as in the proof above. For this assume that the pseudorandom function has two keys, $\kappa_{0}, \kappa_{1}$, and its output for $\mathrm{P}_{i}$ is defined by $\operatorname{PRF}\left(\kappa_{0}, \mathrm{P}_{i}\right) \oplus \kappa_{1}$. Then, receiving $s k_{i}, \omega_{i}, p k_{i}$ from the challenger we can forward the secret key $s k=\left(s k_{0},\left(\kappa_{0}, \kappa_{1}\right)\right)$ for random $\kappa_{0}$ and $\kappa_{1}=$ $\omega_{i} \oplus \operatorname{PRF}\left(\kappa_{0}, \mathrm{P}_{i}\right)$ to the simulated adversary. This choice of $\left(\kappa_{0}, \kappa_{1}\right)$ lets the pseudorandom function map $\mathrm{P}_{i}$ to the desired value $\omega_{i}$ and is identically distributed to a truly random key pair. Hence, if we have an adversary against the certify-then-sign construction for the pseudorandom function, we obtain an adversary against context hiding of one of the underlying schemes as above.

\section{Acknowledgments}

We thank the anonymous reviewers for comments. Marc Fischlin was supported by a Heisenberg grant Fi 940/3-1 of the German Research Foundation (DFG).

\footnotetext{
${ }^{3}$ If we use a pseudorandom function to create the keys on the fly some care must be taken in this step, as discusses after completion of the proof here.
} 


\section{References}

$\left[\mathrm{ABC}^{+} 12\right]$ Jae Hyun Ahn, Dan Boneh, Jan Camenisch, Susan Hohenberger, Abhi Shelat, and Brent Waters. Computing on authenticated data. In Ronald Cramer, editor, TCC 2012: 9th Theory of Cryptography Conference, volume 7194 of Lecture Notes in Computer Science, pages 1-20, Taormina, Sicily, Italy, March 19-21, 2012. Springer, Berlin, Germany. (Cited on pages 1, 2, 3 and 5.)

[ACdMT05] Giuseppe Ateniese, Daniel H. Chou, Breno de Medeiros, and Gene Tsudik. Sanitizable signatures. In ESORICS, volume 3679 of Lecture Notes in Computer Science, pages 159-177. Springer, 2005. (Cited on page1)

[AL11] Nuttapong Attrapadung and Benoît Libert. Homomorphic network coding signatures in the standard model. In Dario Catalano, Nelly Fazio, Rosario Gennaro, and Antonio Nicolosi, editors, PKC 2011: 14th International Workshop on Theory and Practice in Public Key Cryptography, volume 6571 of Lecture Notes in Computer Science, pages 17-34, Taormina, Italy, March 6-9, 2011. Springer, Berlin, Germany. (Cited on page 1)

[ALP12] Nuttapong Attrapadung, Benoit Libert, and Thomas Peters. Computing on authenticated data: New privacy definitions and constructions. In Advances in Cryptology ASIACRYPT 2012, volume 7658 of Lecture Notes in Computer Science, pages 367385. Springer, Berlin, Germany, 2012. (Cited on pages $2,3,4$ and 5 )

$\left[\mathrm{BBD}^{+} 10\right]$ Christina Brzuska, Heike Busch, Özgür Dagdelen, Marc Fischlin, Martin Franz, Stefan Katzenbeisser, Mark Manulis, Cristina Onete, Andreas Peter, Bertram Poettering, and Dominique Schröder. Redactable signatures for tree-structured data: Definitions and constructions. In Jianying Zhou and Moti Yung, editors, ACNS 10: 8th International Conference on Applied Cryptography and Network Security, volume 6123 of Lecture Notes in Computer Science, pages 87-104, Beijing, China, June 22-25, 2010. Springer, Berlin, Germany. (Cited on pages 1 and 5 )

[BF11a] Dan Boneh and David Mandell Freeman. Homomorphic signatures for polynomial functions. In Kenneth G. Paterson, editor, Advances in Cryptology - EUROCRYPT 2011, volume 6632 of Lecture Notes in Computer Science, pages 149-168, Tallinn, Estonia, May 15-19, 2011. Springer, Berlin, Germany. (Cited on page1)

[BF11b] Dan Boneh and David Mandell Freeman. Linearly homomorphic signatures over binary fields and new tools for lattice-based signatures. In Dario Catalano, Nelly Fazio, Rosario Gennaro, and Antonio Nicolosi, editors, PKC 2011: 14th International Workshop on Theory and Practice in Public Key Cryptography, volume 6571 of Lecture Notes in Computer Science, pages 1-16, Taormina, Italy, March 6-9, 2011. Springer, Berlin, Germany. (Cited on page1)

[BFKW09] Dan Boneh, David Freeman, Jonathan Katz, and Brent Waters. Signing a linear subspace: Signature schemes for network coding. In Stanislaw Jarecki and Gene Tsudik, editors, PKC 2009: 12th International Conference on Theory and Practice of Public Key Cryptography, volume 5443 of Lecture Notes in Computer Science, pages 68-87, Irvine, CA, USA, March 18-20, 2009. Springer, Berlin, Germany. (Cited on page 1) 
[BN02] Mihir Bellare and Gregory Neven. Transitive signatures based on factoring and RSA. In Yuliang Zheng, editor, Advances in Cryptology - ASIACRYPT 2002, volume 2501 of Lecture Notes in Computer Science, pages 397-414, Queenstown, New Zealand, December 1-5, 2002. Springer, Berlin, Germany. (Cited on page1)

[CFW12] Dario Catalano, Dario Fiore, and Bogdan Warinschi. Efficient network coding signatures in the standard model. In Marc Fischlin, Johannes Buchmann, and Mark Manulis, editors, PKC 2012: 15th International Workshop on Theory and Practice in Public Key Cryptography, volume 7293 of Lecture Notes in Computer Science, pages 680-696, Darmstadt, Germany, May 21-23, 2012. Springer, Berlin, Germany. (Cited on page 1)

[CH12] Philippe Camacho and Alejandro Hevia. Short transitive signatures for directed trees. In Orr Dunkelman, editor, Topics in Cryptology - CT-RSA 2012, volume 7178 of Lecture Notes in Computer Science, pages 35-50, San Francisco, CA, USA, February 27 - March 2, 2012. Springer, Berlin, Germany. (Cited on page1)

[CJL09] Denis Charles, Kamal Jain, and Kristin Lauter. Signatures for network coding. Int. J. Inf. Coding Theory, 1(1):3-14, March 2009. (Cited on page 1)

[CLX09] Ee-Chien Chang, Chee Liang Lim, and Jia Xu. Short redactable signatures using random trees. In Marc Fischlin, editor, Topics in Cryptology - CT-RSA 2009, volume 5473 of Lecture Notes in Computer Science, pages 133-147, San Francisco, CA, USA, April 20-24, 2009. Springer, Berlin, Germany. (Cited on page 1)

[Des93] Yvo Desmedt. Computer security by redefining what a computer is. In Proceedings on the 1992-1993 workshop on New security paradigms, NSPW '92-93, pages 160-166. ACM, 1993. (Cited on page1)

[Fre12] David Mandell Freeman. Improved security for linearly homomorphic signatures: A generic framework. In Marc Fischlin, Johannes Buchmann, and Mark Manulis, editors, PKC 2012: 15th International Workshop on Theory and Practice in Public Key Cryptography, volume 7293 of Lecture Notes in Computer Science, pages 697-714, Darmstadt, Germany, May 21-23, 2012. Springer, Berlin, Germany. (Cited on page 1)

[GKKR10] Rosario Gennaro, Jonathan Katz, Hugo Krawczyk, and Tal Rabin. Secure network coding over the integers. In Phong Q. Nguyen and David Pointcheval, editors, PKC 2010: 13th International Conference on Theory and Practice of Public Key Cryptography, volume 6056 of Lecture Notes in Computer Science, pages 142-160, Paris, France, May 26-28, 2010. Springer, Berlin, Germany. (Cited on page1)

$\left[\mathrm{HHH}^{+} 08\right]$ Stuart Haber, Yasuo Hatano, Yoshinori Honda, William Horne, Kunihiko Miyazaki, Tomas Sander, Satoru Tezoku, and Danfeng Yao. Efficient signature schemes supporting redaction, pseudonymization, and data deidentification. In Masayuki Abe and Virgil Gligor, editors, ASIACCS 08: 3rd Conference on Computer and Communications Security, pages 353-362, Tokyo, Japan, March 18-20, 2008. ACM Press. (Cited on page 1)

[JMSW02] Robert Johnson, David Molnar, Dawn Xiaodong Song, and David Wagner. Homomorphic signature schemes. In Bart Preneel, editor, Topics in Cryptology - CT-RSA 2002, volume 2271 of Lecture Notes in Computer Science, pages 244-262, San Jose, CA, USA, February 18-22, 2002. Springer, Berlin, Germany. (Cited on page 1) 
[JWL11] Rob Johnson, Leif Walsh, and Michael Lamb. Homomorphic signatures for digital photographs. In George Danezis, editor, FC 2011: 15th International Conference on Financial Cryptography and Data Security, volume 7035 of Lecture Notes in Computer Science, pages 141-157, Gros Islet, St. Lucia, February 28 - March 4, 2011. Springer, Berlin, Germany. (Cited on page 1)

[MR02] Silvio Micali and Ronald L. Rivest. Transitive signature schemes. In Bart Preneel, editor, Topics in Cryptology - CT-RSA 2002, volume 2271 of Lecture Notes in Computer Science, pages 236-243, San Jose, CA, USA, February 18-22, 2002. Springer, Berlin, Germany. (Cited on page 1)

$\left[\mathrm{MSI}^{+} 03\right] \quad$ K. Miyazaki, S. Susaki, M. Iwamura, T. Matsumoto, R. Sasaki, and H. Yoshiura. Digital documents sanitizing problem. In Technical Report ISEC2003-20. IEICE, 2003. (Cited on page 1)

[NTKK09] Ryo Nojima, Jin Tamura, Youki Kadobayashi, and Hiroaki Kikuchi. A storage efficient redactable signature in the standard model. In Pierangela Samarati, Moti Yung, Fabio Martinelli, and Claudio Agostino Ardagna, editors, ISC 2009: 12th International Conference on Information Security, volume 5735 of Lecture Notes in Computer Science, pages 326-337, Pisa, Italy, September 7-9, 2009. Springer, Berlin, Germany. (Cited on page 1)

[SBZ01] Ron Steinfeld, Laurence Bull, and Yuliang Zheng. Content extraction signatures. In ICISC, volume 2288 of Lecture Notes in Computer Science, pages 285-304. Springer, 2001. (Cited on page 1)

[SSM04] Siamak Fayyaz Shahandashti, Mahmoud Salmasizadeh, and Javad Mohajeri. A provably secure short transitive signature scheme from bilinear group pairs. In Carlo Blundo and Stelvio Cimato, editors, SCN 04: 4th International Conference on Security in Communication Networks, volume 3352 of Lecture Notes in Computer Science, pages 60-76, Amalfi, Italy, September 8-10, 2004. Springer, Berlin, Germany. (Cited on page 1)

[WCZ ${ }^{+}$07] Licheng Wang, Zhenfu Cao, Shihui Zheng, Xiaofang Huang, and Yixian Yang. Transitive signatures from braid groups. In K. Srinathan, C. Pandu Rangan, and Moti Yung, editors, Progress in Cryptology - INDOCRYPT 200\%: 8th International Conference in Cryptology in India, volume 4859 of Lecture Notes in Computer Science, pages 183196, Chennai, India, December 9-13, 2007. Springer, Berlin, Germany. (Cited on page1)

[Yi07] Xun Yi. Directed transitive signature scheme. In Masayuki Abe, editor, Topics in Cryptology - CT-RSA 2007, volume 4377 of Lecture Notes in Computer Science, pages 129-144, San Francisco, CA, USA, February 5-9, 2007. Springer, Berlin, Germany. (Cited on page 1) 